\title{
EFFICIENCY OF NANO SILVER FLUORIDE ON PREVENTION OF DENTAL CARIES ON INTACT ENAMEL SURFACE ASSESSED BY EDX-ANALYSIS (IN VITRO STUDY)
}

\author{
Ayman Sabbah* and Inas Helwa**
}

\begin{abstract}
Aim: The purpose of this study was to evaluate the preventive effect of nanosilver fluoride (NSF) on intact enamel surface by using energy dispersive x-ray spectroscopy (EDX-analysis) to detect the superficial fluoride precipitation on the surface of intact human dental enamel.

Methodology: In this study, we used energy-dispersive X-ray spectroscopy (EDX) to detect the precipitation of fluoride on the surface of flat polished enamel surface after NSF application.

Results: Our data has shown that treatment with NSF for 2 minutes led to significant precipitation of fluoride on enamel surface as compared to untreated samples. The precipitated fluoride was detected 7 days after the varnish application.

Conclusion: Nanosilver fluoride varnish application on the surface of sound unaffected enamel can be an effective caries preventive measure. Fluoride precipitation on the surface of enamel and its further incorporation in the crystal lattice will lead to the formation of less soluble fluorapatite crystals that are more resistant to the cariogenic bacterial demineralizing acids.
\end{abstract}

\section{INTRODUCTION}

Dental caries remains the most prevalent oral disease worldwide. It is a global public health problem that affects people in both developed and developing countries. Fermentation of carbohydrates by the cariogenic bacteria located in the dental plaque results in organic acids that leads to demineralization of enamel and dentin. ${ }^{(1)}$ The pathogenesis of dental caries is dynamic and involves periods of demineralization and remineralization which may be reversible if detected at. an early stage. ${ }^{(2)}$ The management of dental caries includes conservative and preventive methods that require early diagnosis to preserve the tooth structure by either arresting caries at an early stage or preventing the initial demineralization process. ${ }^{(2-5)}$

* Lecturer of Pediatric Dentistry, Pediatric Dentistry and Orthodontic Department, Faculty of Oral and Dental medicine, Misr International University

** Lecturer of Oral Biology, Histopathology Department, Faculty of Oral and Dental medicine, Misr International University 
To date, topical fluorides are considered the most effective method for the prevention of caries occurrence and the arrest of early carious lesions. $(4,6,7)$ Sodium fluoride varnishes are widely used due to the ease and rapid application as well as fast setting on the tooth surface minimizing the common drawbacks of topical fluoride application including swallowing or gagging that may lead to toxicity or dental fluorosis when especially in young children. ${ }^{\left({ }^{8}\right)}$ When the hydroxyl ions in the enamel crystal lattice structure get partially replaced by fluoride ions, the resulting fluoroapatite or calcium fluoride crystals are more resistant to lowering of the $\mathrm{pH}$ caused by the acidic bacteria. This crystal lattice stability yields the enamel surface less prone to dissolution by the microbial demineralizing acids and thus has a protective effect against caries. ${ }^{(9)}$

Silver has been utilized in dental field for decreasing caries incidence in both primary and permanent dentitions due to broad spectrum antimicrobial activity, lower toxicity and lack of bacterial resistance. ${ }^{\left({ }^{10}\right)}$ Currently, nanosilver fluoride (NSF) is considered an alternate to silver diamine fluoride (SDF) regarding caries prevention ${ }^{(11,12)}$, but without he common SDF disadvantages including staining of the teeth porous tissues and restorations.

The superior properties of nanosilver fluoride may be attributed to the nanoparticle size of silver. (13) Silver nanoparticles are added to orthodontic brackets and adhesive materials in an attempt to prevent caries that commonly affects teeth during orthodontic treatment. It can also be used as a base for various restorative materials to prevent the occurrence of secondary caries. (14) Many studies have previously discussed the role of silver nanomaterials in caries prevention. ${ }^{(15)}$ However, for the time being no studies have been done to evaluate the preventive effect of NSF on intact enamel of permanent anterior teeth. Therefore, the aim of this in vitro study is to assess the effect of nanosilver on prevention of dental caries fluoride by detecting the superficial fluoride precipitation on the surface of intact human dental enamel using EDX following a single two-minute NSF treatment.

\section{MATERIALS AND METHODS}

\section{Materials}

Artificial saliva was prepared in the Faculty of pharmacy, Misr International University and as previously described by Abo El Soud et al. ${ }^{(1)}$ Briefly, the artificial saliva was prepared from $1.5 \mathrm{mmol} / \mathrm{L}$ $\mathrm{CaCl}_{2}, 50 \mathrm{mmol} / \mathrm{L} \mathrm{KCl}, 0.9 \mathrm{mmol} / \mathrm{L} \mathrm{KH}_{2} \mathrm{PO}_{4}$ and adjusted to a $\mathrm{pH}$ of 7.3. Nanosilver fluoride varnish was prepared by NanoGate company, Cairo, Egypt. The size of nanosilver particles of this preparation was approximately $10 \mathrm{~nm}$. The concentration of fluoride and silver nitrate was 10147 ppm and 400 ppm respectively.

\section{Samples}

We used twenty de-identified permanent incisors that were extracted for reasons other than dental decay, such as severe mobility due to irreversible periodontitis. Our inclusion criteria encompassed any permanent incisor that has never been restored and showed intact enamel surface that is free of caries, white spots, cracks, any visible anomalies or signs of demineralization. The selected teeth were initially polished to be free of debris and minimize inter-individual differences among teeth as recommended by Scolz et al. ${ }^{(16)}$ We randomly divided our specimens into two groups $(\mathrm{n}=10)$.

\section{Group 1: (Negative Control Group)}

This group included ten incisors that were left untreated (negative control). Teeth were stored in a tightly capped container filled with artificial saliva for seven days at $37^{\circ} \mathrm{C}$. After the seven days, teeth were removed from the artificial saliva, washed with deionized water for approximately 30 seconds, then lightly dried with cotton swabs for another 30 seconds. Before EM visualization, specimens 
were thoroughly inspected for any signs of demineralization to make sure that enamel surface of all teeth were visibly intact.

\section{Group 2: (NSF varnish application)}

This group included ten incisors that were treated with nanosilver fluoride varnish using cotton swabs for two minutes on the labial surface. Samples were then washed with deionized water for approximately 30 seconds, then lightly dried with cotton swabs for another 30 seconds. Specimens were then incubated in a tightly capped container filled with artificial saliva for seven days at $37^{\circ}$.

\section{Methods of assessment}

\section{Scanning electron microscopy}

All samples were removed from the artificial saliva after seven days, and left for two hours to dry at room temperature before the analysis. The specimens were examined using scanning electron microscope Zeiss Sigma 300 VP at the Egyptian Petroleum Research Institute (EPRI), Cairo, Egypt to observe the enamel surface ultra-morphology.

\section{Energy dispersive $x$-ray spectroscopy: (EDX-analysis)}

We used energy dispersive x-ray spectroscopy to measure the mineral content of the specimens (Calcium, phosphorous and fluoride) as atomic percentage (atomic \%).

\section{Statistical analysis}

Numerical data were explored for normality by checking the distribution of data and using tests of normality (Kolmogorov-Smirnov and Shapiro-Wilk tests). Data showed normal (parametric) distribution. Data were presented as mean and standard deviation (SD) values. Student's t-test was used to compare between the two groups. The significance level was set at $\mathrm{P} \leq 0.05$. Statistical analysis was performed with IBM SPSS Statistics for Windows, Version 23.0. Armonk, NY: IBM Corp.

\section{RESULTS}

\section{Scanning electron microscopy (SEM):}

SEM evaluation of group 1 (Negative control group) revealed enamel rods with inter rod materials on a smooth surface free of cracks and debris (Fig. 1A). Examination of group 2 specimens treated with NSF varnish revealed precipitation of NSF particles on the surface of enamel (Fig. 1B) suggesting a potential protective effect of fluoride on the enamel surface.

\section{Energy dispersive x-ray spectroscopy (EDX):}

\section{Fluoride}

As shown in table 1 and (Fig. 2): NSF varnish group showed statistically significantly higher mean Fluoride weight $\%$ than negative control group $(P$-value $<0.001$, Effect size $=2.165)$.

\section{Calcium}

As shown in table 1 and (Fig. 2): NSF varnish group showed statistically significantly higher mean Calcium weight $\%$ than negative control group $(P$-value $<0.001$, Effect size $=1.794)$.

\section{Phosphorus}

As shown in table 1 and (Fig. 2): NSF varnish group showed statistically significantly lower mean Phosphorus weight $\%$ than negative control group $(P$-value $<0.001$, Effect size $=2.205)$.

\section{Calcium: Phosphorus ratio}

As shown in table 1 and (Fig.3): NSF varnish group showed statistically significantly higher mean Calcium: Phosphorus ratio than negative control group $(P$-value $<0.001$, Effect size $=2.726)$. 
TABLE (1): Descriptive statistics and results of Student's t-test for comparison between minerals weight $\%$ in the two groups

\begin{tabular}{ccccccc}
\hline & \multicolumn{2}{c}{$\begin{array}{c}\text { NSF varnish } \\
(\mathrm{n}=10)\end{array}$} & \multicolumn{2}{c}{$\begin{array}{c}\text { Negative control } \\
(\mathrm{n}=10)\end{array}$} & P-value & Effect size $(d)$ \\
\cline { 2 - 6 } Mineral & Mean & SD & Mean & SD & & 2.165 \\
\hline NSF varnish & 8.03 & 2.01 & 4.6 & 1 & $<0.001 *$ & 1.794 \\
\hline Calcium & 55.59 & 3.38 & 50.8 & 1.68 & $0.001^{*}$ & 2.205 \\
\hline Phosphorus & 35.66 & 2.95 & 43.3 & 3.91 & $<0.001 *$ & $<0.001^{*}$ \\
\hline
\end{tabular}

*: Significant at $P \leq 0.05$

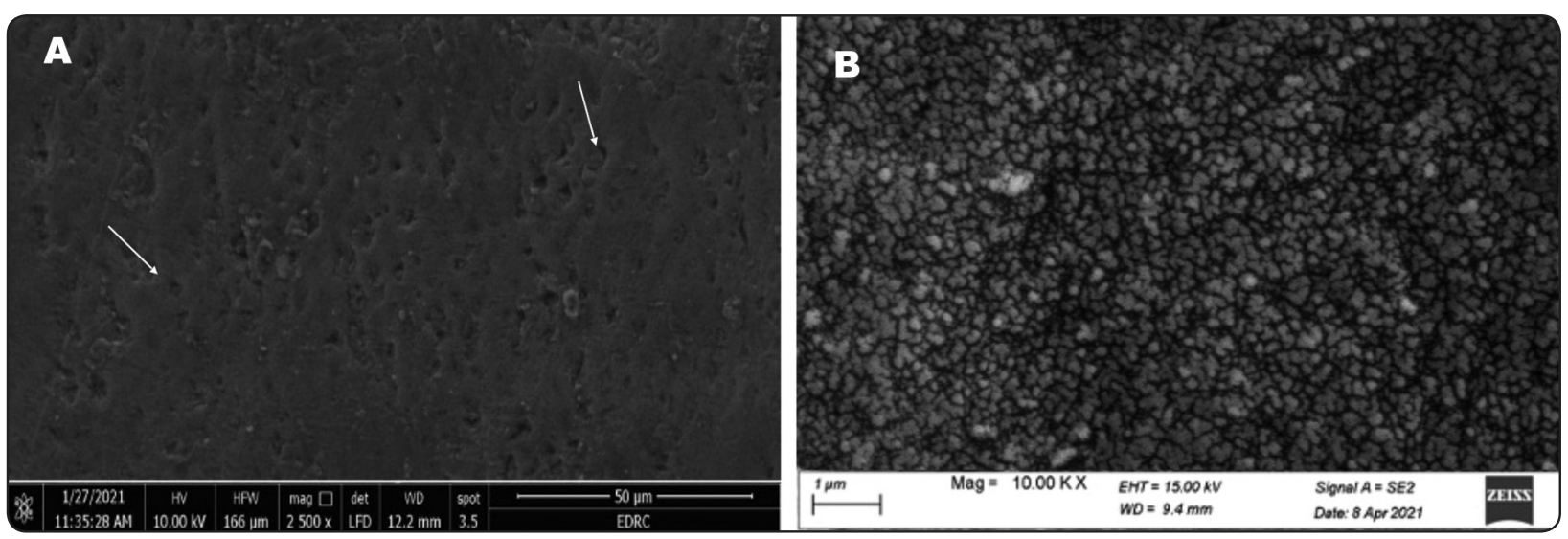

Fig. (1) SEM photographs. (A) Group 1 (negative control) showing arranged enamel rods and inter rod material (arrow heads). (B) Group 2 (NSF treatment) showing fluoride precipitation of NSF particles on the surface of enamel. (Magnification 2500X and $10000 \mathrm{X}$ respectively).

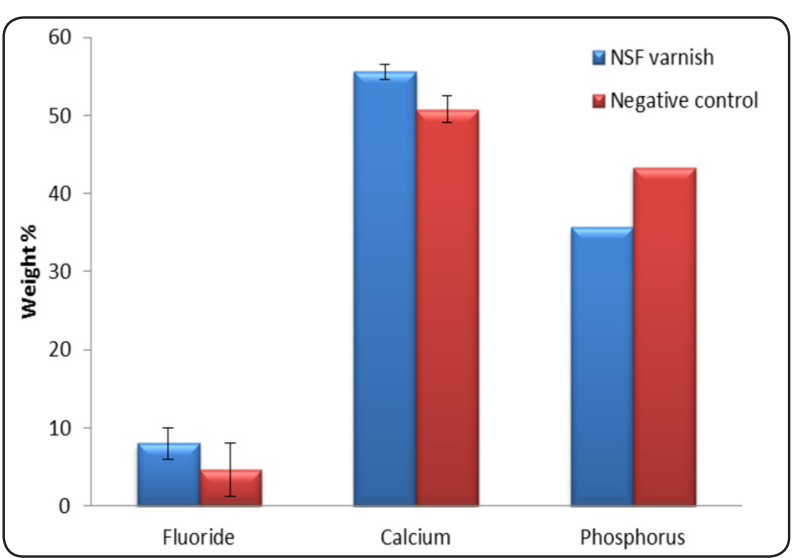

Fig. (2) Bar chart representing mean and standard deviation values for minerals weight $\%$ in the two groups

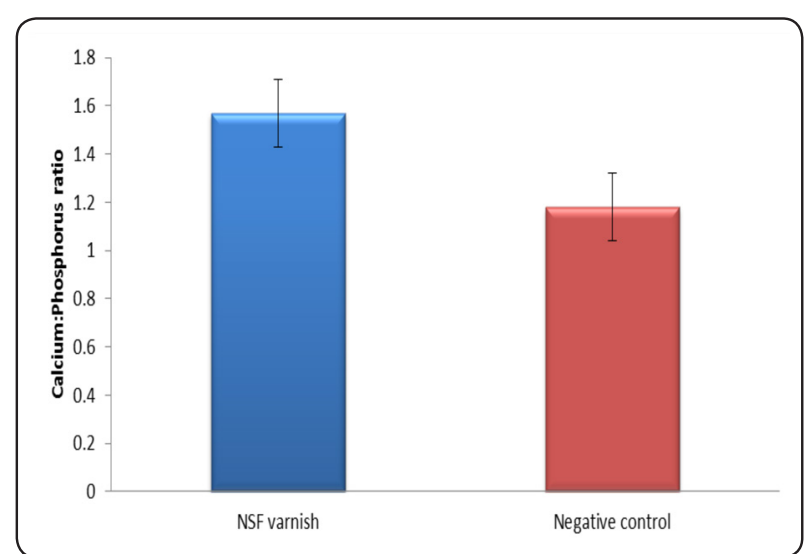

Fig. (3). Bar chart representing mean and standard deviation values for Calcium:Phosphorus ratios in the two groups 


\section{DISCUSSION}

The current study aims at exploring the precipitation of nanosilver fluoride (NSF) on the surface of enamel after NSF varnish application for two minutes. In this in vitro study, we used energydispersive X-ray spectroscopy (EDX) to detect fluoride precipitation on the surface of enamel after applying a thin layer of NSF varnish (10147 ppm) for two minutes. We measured the weight percent (Wt\%) of Calcium, phosphorous and fluoride before and after the varnish application, followed by one week of incubation in artificial saliva. All fluoridated specimens showed approximately twofold increase in the $\mathrm{Wt} \%$ of fluoride following the varnish application for two minutes, followed by the seven-days incubation in artificial saliva. There was almost no change in the $\mathrm{Wt} \%$ of calcium and phosphorous. Scanning electron microscopy images of the treated specimens revealed precipitation of the fluoride particles on the enamel surface as compared to the non-treated control samples that were only incubated in artificial saliva.

For decades, fluoride has been considered a major contributor and a cornerstone of the decline of dental caries incidence across populations. Amongst various oral conditions, dental caries stands out as the most prevalent condition with a $35 \%$ prevalence (for all age groups combined) according to the Global Burden of Disease (GBD) 2010 study ${ }^{(3)}$. According to the CDC, in 2014 the prevalence of caries among adults (20-64 years) in the US was 92\%. ${ }^{(17)}$ This percentage has declined by 2\% from 2004 to 2019. On the other hand, the prevalence of dental caries among children (6-8 years) in 2019 was 52\% with no decline detected since 2004. ${ }^{(18)}$ Topical fluorides in the form of gel, varnish, toothpaste and mouth wash have a significant effect in reducing dental caries occurrence among children. ${ }^{(19,20)}$ The overall prevalence of dental caries in developing countries is comparatively high and varies according to the socio-economic status. (21) Low socio-economic status seems to be a predisposing factor to caries incidence. ${ }^{(22)}$ Even in the US and other developed countries, dental caries continues to be a major chronic disease of childhood. ${ }^{(23)}$

A previous cross-sectional study conducted among a group of Egyptian children and published in 2021, has shown that some children suffered from distinct visual changes in enamel only without breakdown according to the Caries Assessment Spectrum and Treatment index system (CAST). These carious lesions could have been prevented or reversibly and non-invasively managed by fluoride which has a preventive and remineralizing effect on hard tooth structure ${ }^{(24)}$. With the emergence of nanotechnology as a promising armamentarium of the $21^{\text {st }}$ century, its applications in the dental field have been extensively growing. The use of silver nanoparticles in dentistry has gained much attention for its anti-bacterial mechanism and its potential to counteract the drawbacks of silver diamine fluorides (SDF) such as tooth discoloration and gingival inflammation. ${ }^{(1,2,4,25)}$ The effect of topical fluorides on enamel includes enhancing enamel remineralization and preventing demineralization. Previous studies have shown that the presence of fluorides in a solution surrounding the enamel crystals yields them more resistant to acidic challenges. ${ }^{(26)}$ Our data have shown that fluoride has precipitated on the surface of sound enamel, which will increase the enamel surface resistance to acidic environment that promotes dental caries, in addition the silver nanoparticles have a direct antibacterial effect that makes this formula more protective to the hard tooth structure.

This result agrees with previously published data by $\mathrm{Wu}$ and et al. which showed that treating enamel surfaces with artificial carious lesions by silver nanomaterials reduces the lesion depth as compared to those treated by water following biofilm challenge. ${ }^{(27)}$ Scarpelli et al. stated that the microhardness of enamel surface with artificial 
caries can be increased by treatment of silver nanoparticles (2), and Nozari et al. mentioned that treatment of enamel caries by nanosilver fluoride showed higher microhardness than those treated by sodium fluoride ${ }^{(28)}$, which supports the significance of using nanosilver fluoride as a preventive measure for dental caries. On the other hand, Vieira Costa e Silva et al. and Teixeira et al. proved that the microhardness value of sound enamel surface treated by nanosilver fluoride is similar to that treated with sodium fluoride. ${ }^{(29,30)}$ Furthermore, two separate clinical trials by Tirupathi et al. and Dos Santos et al. concluded that nanosilver fluoride has no effect on arresting the dentin caries in children. ${ }^{(31,32)}$ Accordingly, further clinical studies are needed to accurately assess the nanosilver fluoride as a preventive measure.

\section{CONCLUSION}

Topical application of NSF varnish can be a successful and cost-effective caries-preventive measure for children. NSF overcomes the known disadvantages of SDF such as tooth discoloration. Further clinical studies will be necessary to examine the caries reduction rate following NSF application.

\section{REFERENCES}

1. Abo El Soud, A., Elsaied, H., Omar S. Comparative Evaluation of the Comparative Evaluation of the Effects of Silver Diamine Fluoride (Commercial and Lab Prepared) versus Nano silver fluoride on Demineralized Human Enamel Surfaces (In Vitro Study). E D J. 2020; 66(1):15363

2. Scarpelli BB, Punhagui MF, Hoeppner MG, Almeida RSC, Juliani FA, Guiraldo RD, Berger SB. In Vitro Evaluation of the Remineralizing Potential and Antimicrobial Activity of a Cariostatic Agent with Silver Nanoparticles. Braz Dent J. 2017 ;28(6):738-43. doi: 10.1590/0103-6440201701365

3. Featherstone JDB, Crystal YO, Alston P, Chaffee BW, Doméjean S, Rechmann P, Zhan L and Ramos-Gomez. Evidence-Based Caries Management for All Ages-Practical Guidelines. Front. Oral. Health. 2021; 2021:657518. doi: 10.3389 /froh.2021.657518
4. Butrón Téllez Girón C, Hernández Sierra JF, DeAlbaMontero I, Urbano Peña MLA, Ruiz F. Therapeutic Use of Silver Nanoparticles in the Prevention and Arrest of Dental Caries. Bioinorg Chem Appl. 2020; 2020:8882930. doi: $10.1155 / 2020 / 8882930$

5. Fontana M, González-Cabezas C. Noninvasive Caries Risk-based Management in Private Practice Settings May Lead to Reduced Caries Experience Over Time. J Evid Based Dent Pract. 2016 ;16(4):239-42. doi: 10.1016/j. jebdp.2016.11.003

6. Amaechi BT, AbdulAzees PA, Alshareif DO, Shehata MA, Lima PPCS, Abdollahi A, Kalkhorani PS, Evans V. Comparative efficacy of a hydroxyapatite and a fluoride toothpaste for prevention and remineralization of dental caries in children. BDJ Open. 2019; 5:18. doi: 10.1038/ s41405-019-0026-8

7. Pitts NB, Zero DT, Marsh PD, Ekstrand K, Weintraub JA, Ramos-Gomez F, Tagami J, Twetman S, Tsakos G, Ismail A. Dental caries. Nat Rev Dis Primers. 2017 ;2017:17030. doi: $10.1038 /$ nrdp. 2017.30

8. Chu $\mathrm{CH}$, Lo E. Uses of sodium fluoride varnish in dental practice. Ann R Australas Coll Dent Surg. 2008 ;19:58-61

9. Pajor K, Pajchel L, Kolmas J. Hydroxyapatite and Fluorapatite in Conservative Dentistry and Oral Implantology- A Review. Materials (Basel). 2019 ; 2019: 2683. doi: $10.3390 / \mathrm{ma} 12172683$

10. Fakhruddin KS, Egusa H, Ngo HC, Panduwawala C, Pesee S, Samaranayake LP. Clinical efficacy and the antimicrobial potential of silver formulations in arresting dental caries: A systematic review. BMC Oral Health. 2020 ;20(1):1-13. https://doi.org/10.1186/s12903-020-01133-3

11. Nanda K J, Naik S. An In-Vitro Comparative Evaluation of Pre-treatment With Nano-Silver Fluoride on Inhibiting Secondary Caries at Tooth Restoration Interface. Cureus. 2020; 2020:7934. doi:10.7759/cureus.7934

12. Targino AG, Flores MA, dos Santos Junior VE, de Godoy Bené Bezerra F, de Luna Freire H, Galembeck A, Rosenblatt A. An innovative approach to treating dental decay in children. A new anti-caries agent. J Mater Sci Mater Med. 2014 ;25(8):2041-7. doi: 10.1007/s 10856-014-5221-5

13. Dos Santos VE, Filho AV, Ribeiro Targino AG, Pelagio Flores MA, Galembeck A, Caldas AF, et al. A new "silverBullet" to treat caries in children - Nano Silver Fluoride: A randomised clinical trial. J Dent. 2014; 42(8):945-51. doi:10.1016/j.jdent.2014.05.017 
14. Metin-Gürsoy G, Taner L, Akca G. Nanosilver coated orthodontic brackets: in vivo antibacterial properties and ion release. Eur J Orthod. 2017 ;39(1):9-16. doi: 10.1093/ ejo/cjv097

15. Xiao S, Liang K, Weir MD, Cheng L, Liu H,3 Zhou X et al. Combining Bioactive Multifunctional Dental Composite with PAMAM for Root Dentin Remineralization. Materials (Basel). 2017;10(1):89. doi:10.3390/ma10010089

16. Scholz KJ, Federlin M, Hiller KA, Ebensberger H, Ferstl G, Buchalla W. EDX-analysis of fluoride precipitation on human enamel. Sci Rep. 2019; 2019:13442. https://doi. org/10.1038/s41598-019-49742-5

17. Centers for Disease Control and Prevention. Dental caries among adults and younger adults [Internet]. Dental cariees among adults and younger adults. 2019 [cited 2021 Aug 4]. Available from: https://www.cdc.gov/oralhealth/publications/OHSR-2019-dental-carries-adults.html\#Aged20

18. Centers for Disease Control and Prevention. Dental Caries in Primary Teeth [Internet]. Dental Caries in Primary Teeth. 2019 [cited 2021 Aug 4]. Available from: https://www.cdc. gov/oralhealth/publications/OHSR-2019-dental-cariesprimary-teeth.html

19. Marinho VC, Higgins JP, Logan S, Sheiham A. Topical fluoride (toothpastes, mouthrinses, gels or varnishes) for preventing dental caries in children and adolescents. Cochrane Database Syst Rev. 2003 ;2003:CD002782. doi: 10.1002/14651858.CD002782

20. Teshome A, Muche A, Girma B. Prevalence of Dental Caries and Associated Factors in East Africa, 20002020: Systematic Review and Meta-Analysis. Front Public Health. 2021 ; 2021(9): 645091. doi: 10.3389/ fpubh.2021.645091.

21. Marcenes W, Kassebaum NJ, Bernabé E, Flaxman A, Naghavi M, Lopez A, et al. Global burden of oral conditions in 1990-2010: A systematic analysis. J Dent Res. 2013; 92(7):592-7. doi:10.1177/0022034513490168

22. Knoblauch U, Ritschel G, Weidner K, et al. The association between socioeconomic status, psychopathological symptom burden in mothers, and early childhood caries of their children. PLoS One. $2019 ; 2019: 0224509$. doi:10.1371/journal.pone.0224509

23. Clark MB, Keels MA, Slayton RL; SECTION ON ORAL HEALTH. Fluoride Use in Caries Prevention in the Primary Care Setting. Pediatrics. 2020 ;2020:2020034637. doi: $10.1542 /$ peds.2020-034637
24. Hamza, A., Abd Al Gawad, R., Hanafy, R. Prevalence of Dental Caries Among a Group of Egyptian Children Using Caries Assessment Spectrum and Treatment Index:A Cross Sectional Study. Adv Dent J. 2021; 3(2): 63-72. doi: 10.21608/adjc.2021.51736.1086

25. Nagireddy VR, Reddy D, Kondamadugu S, Puppala N, Mareddy A, Chris A. Nanosilver Fluoride-A Paradigm Shift for Arrest in Dental Caries in Primary Teeth of Schoolchildren: A Randomized Controlled Clinical Trial. Int J Clin Pediatr Dent. 2019; 12(6):484-90. doi:10.5005/ jp-journals-10005-1703

26. Mohammed NR, Lynch RJ, Anderson P. Effects of fluoride concentration on enamel demineralization kinetics in vitro. J Dent. 2014 ;42(5):613-8. doi: 10.1016/j. jdent.2013.12.005

27. Wu R, Zhao Q, Lu S, Fu Y, Yu D, Zhao W. Inhibitory effect of reduced graphene oxide-silver nanocomposite on progression of artificial enamel caries. J Appl Oral Sci. 2018; 2018:20180042. doi:10.1590/1678-7757-2018-0042

28. Nozari A, Ajami S, Rafiei A, Niazi E. Impact of Nano Hydroxyapatite, Nano Silver Fluoride and Sodium Fluoride Varnish on Primary Teeth Enamel Remineralization: An In Vitro Study. J Clin Diagn Res. 2017 ;11(9):97-100. doi: 10.7860/JCDR/2017/30108.10694

29. Vieira Costa e Silva A, Teixeira J, Mota C, Clayton Cabral Correia Lins E, Correia de Melo Júnior P, de Souza Lima M, et al. In Vitro morphological, optical and microbiological evaluation of nanosilver fluoride in the remineralization of deciduous teeth enamel. Nanotech Rev. 2018 ;7(6): 50920. https://doi.org/10.1515/ntrev-2018-0083

30. Ribeiro Targino AG, Galembeck A, Rosenblatt A. Effects of a New Nano-Silver Fluoride-Containing Dentifrice on Demineralization of Enamel and Streptococcus mutans Adhesion and Acidogenicity. Int J Dent. 2018 ; 2018:1351925. doi: 10.1155/2018/1351925

31. Tirupathi S, Svsg N, Rajasekhar S, Nuvvula S. Comparative cariostatic efficacy of a novel Nano-silver fluoride varnish with $38 \%$ silver diamine fluoride varnish a double-blind randomized clinical trial. J Clin Exp Dent. 2019 ;11(2):105-12. doi: 10.4317/jced.54995

32. Dos Santos Jr VE, Filho AV, Ribeiro Targino AG, Pelagio Flores MA, Galembeck A, Caldas Jr AF et al. A new "silverbullet" to treat caries in children-nano silver fluoride: a randomised clinical trial. J Dent. 2014; 42(8):945-51. doi:10.1016/j.jdent.2014.05.017 